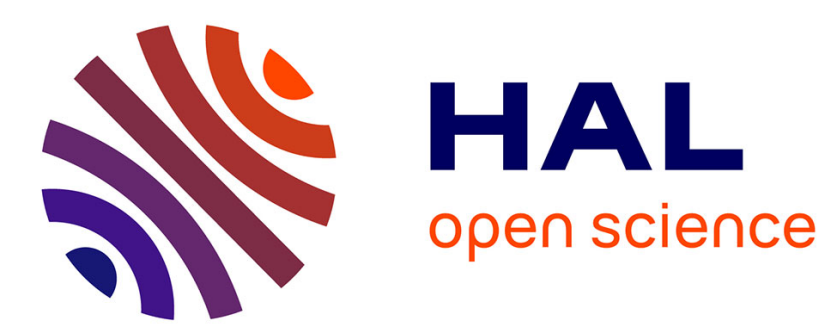

\title{
Online Transfer Learning and Organic Computing for Deep Space Research and Astronomy
}

\author{
Sadanandan Natarajan
}

\section{To cite this version:}

Sadanandan Natarajan. Online Transfer Learning and Organic Computing for Deep Space Research and Astronomy. 2019. hal-02061485

\section{HAL Id: hal-02061485 \\ https://hal.science/hal-02061485}

Preprint submitted on 8 Mar 2019

HAL is a multi-disciplinary open access archive for the deposit and dissemination of scientific research documents, whether they are published or not. The documents may come from teaching and research institutions in France or abroad, or from public or private research centers.
L'archive ouverte pluridisciplinaire HAL, est destinée au dépôt et à la diffusion de documents scientifiques de niveau recherche, publiés ou non, émanant des établissements d'enseignement et de recherche français ou étrangers, des laboratoires publics ou privés. 


\title{
Online Transfer Learning and Organic Computing for Deep Space Research and Astronomy
}

\author{
Sadanandan Natarajan \\ Faculty of Computer Science and Mathematics \\ University of Passau \\ Passau, Germany \\ natara01@gw.uni-passau.de
}

\begin{abstract}
Deep space exploration is the pillars within the field of outer space analysis and physical science. The amount of knowledge from numerous space vehicle and satellites orbiting the world of study are increasing day by day. This information collected from numerous experiences of the advanced space missions is huge. These information helps us to enhance current space knowledge and the experiences can be converted and transformed into segregated knowledge which helps us to explore and understand the realms of the deep space.. Online Transfer Learning (OTL) is a machine learning concept in which the knowledge gets transferred between the source domain and target domain in real time, in order to help train a classifier of the target domain. Online transfer learning can be an efficient method for transferring experiences and data gained from the space analysis data to a new learning task and can also routinely update the knowledge as the task evolves.

Index Terms-Transfer Learning, Online Transfer Learning, Organic Computing, Deep Space Research, Outer Space Explorations
\end{abstract}

\section{INTRODUCTION}

Deep space research is one of the highly discussed topics in science for mankind survival and settlement in planets and space outside planet Earth. It can explain the ever-evolving domain as the exploration of distant regions of outer space, where physical exploration of space can be conducted by both human spaceflights and robotic spacecraft for the mankind space survival.

Mankind explored the deep space with the help of enormous advancement in computer technology. The outer space now becomes a gigantic dataset for astronomers that can be used for various purposes like, predict the cosmological effects, planet classification, etc. One such way, astronomers use these data for morphological classification of galaxies, whose principal goal is to obtain an insight into galaxy formation and evolution. Galaxy classification requires human intervention as the data received by the spacecraft were photographic plates and requires visual inspection and analysis. But as more and more data are produced, it has become unrealistic and impossible to devote human resources to the time-consuming and expensive process of galaxy, planet or star classification. With machine learning, major space companies like NASA now automate image analysis through a neural network and data mining algorithms.

Similarly, advances in data analysis had not matched the technological advances for classification and thus creates a massive imbalance between the rates at which the data being collected and being processed. Machine learning offers a way to correct this imbalance by computing the image analysis in fraction of seconds. It is also used to process all data from the satellite sensors and the mission control input and convert it into knowledge. It is time consuming and inefficient to create models for specific tasks from scratch whereas, it is easier if the model adapts itself and helps apply the existing knowledge to a new task with minimal input. It is also impractical to rebuild a model every time a spacecraft encounters a new obstacle. Another issue for any machine learning task is the lack of availability of large annotated data sets of adequate quality to build highly precise models.

Online transfer learning is a machine learning framework where the knowledge gets transferred from the source domain to target Domain in real time and helps training a classifier of the target domain. This reduces the time and complexity and proven to be a cost-effective solution. The data distribution of the source domain and target domain can be very different. Organic computing aims at mastering the complexity in a technical system by equipping technical systems with life-like properties, by characteristics observed In natural systems such as self-learning and self-organization. The advantage of online transfer learning is that it continuously updates the model with new data.

The combination of OTL with organic computing yields a high result in space for machine learning models to learn and assimilate the knowledge and it can extrapolate the existing knowledge to various tasks. These methods will help solve new tasks and also learn from new experiences to enhance future capabilities for space programs.

This paper is a survey of different methods of OTL that can be used in deep space research, organic computing methods to enhance the space experiences and they can enhance current methods in deep space using the proposed OTL methods.

The rest of the paper is organized is as follows. The section (II) explains the related work of various researchers in Machine Learning and Outer Space. The section (III) discuss the current methods used in deep space research. The section (IV) explains about the concept of online transfer learning and organic computing and its practical use case in day-today outer space research scenarios. Finally, the section (V) summarizes the current methods and enhanced method with 
the concept of OTL and organic computing of this paper.

\section{RELATED WORK}

Many advancements evolved in space research. Autonomous agents are used to observe the environment and make independent decisions about which actions to take, what data to collect and what to transmit back to earth. [13] explained an important method that features how semantic abilities learned by Convolutional Neural Networks (CNN) can be transferred. They were motivated by the absence of perfectly annotated large datasets from the deep space research observations. Space research can be classified into various types of chronology and domains and each domain are different from one another. Machine learning implementation in this domain requires large-scale adaptation of the cross-domains to efficiently extract the data labels to the target learning domain. [11] Shows how ML models can combine multiple cross-domains classifications to extract the data and knowledge into the target domain.

[1] Reveals a specific use case of transfer learning in space research for multiple spacecraft scenarios. It provides an indepth analysis of the classification of feature vectors associated with spacecraft and provides some analysis of such systems. It also discusses the orbit determination of multiple spacecraft scenarios with distributed regression and transfer learning. The model represents orbit determination as a learning problem involving distributed regression.

Semantic segmentation plays a very important role in understanding and interpreting a scene, we can use semantic segmentation in space research for detecting inter-galactic activities and enhance their knowledge. [20] considers an ensemble model incorporating knowledge transfer based on drones for the semantic segmentation of aerial images. The Passive Aggressive(PA) algorithm is popular for the online update of models. [16] discuss a family of algorithms for online learning predictions. The algorithm is used for different outcome scenarios at different settings. PA can be used to predict highly non-linear functions by implementing a kernel trick.

\section{Current Methods in-use in Deep Space RESEARCH AND MISSIONS}

[28] Some current machine learning methods used in space research and exploration are $\mathrm{CNN}$, reinforcement learning, Support Vector Machines(SVM), Naive Bayes, etc. Though some of the above methods are similar to the proposed methods like Reinforcement Learning and CNN, their method implementation and usage is different. The current methods use homogeneous domain selection and have critical drawbacks. Let us see the methods implementation and drawbacks in some of the deep space ML implementation area.

\section{A. Convolutional Neural Networks in Deep Galaxy Classi- fication}

Deep galaxies architecture consists of 8 layers, and the one main convolutional layer for features extraction with 96 filters, followed by two principles fully connected layers

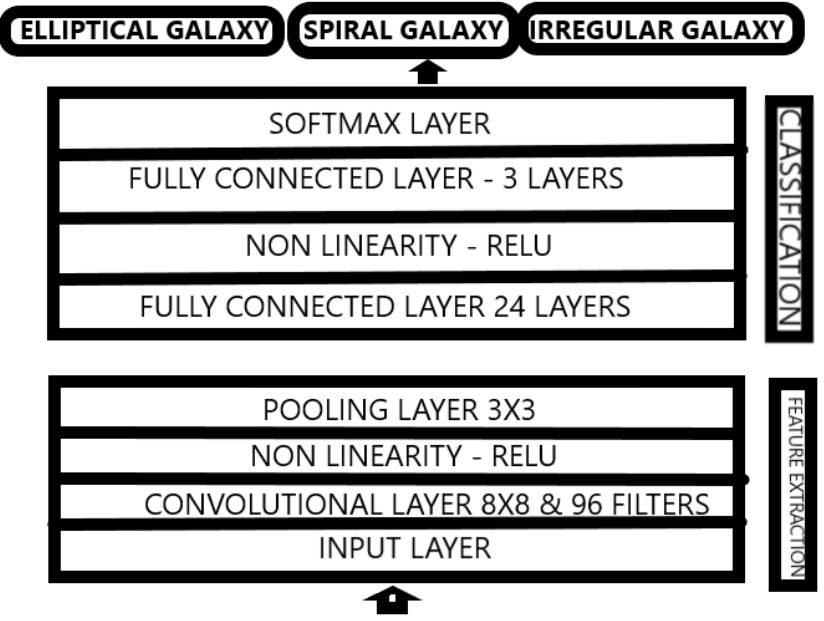

Figure 1. Convolutional Layers in Galaxy Classification [13

for classification. [13] proposed a concept where a deep Neural network architecture for galaxy can be implemented. The author visualizes the proposed architecture in a graphical representation. The concept has 8 layers in which the main convolutional layer is used for features extraction. It is followed by two fully connected layers for further classification. The first layer is the input layer. The second layer is the convolution layer. The third layer is a Rectified Linear Unit (RELU) is which used as the nonlinear activation function. In the fourth layer, Pooling is performed with subsampling.In layer number five, each fully connected layer consists of 24 neurons with RELU activation function. The last fully connected layer have only three neurons and it uses a soft-max layer to obtain class memberships for visualizing the feature extraction in the proposed deep neural architecture. Figure.1 shows the different images resulted from applying the first convolution layer with 96 filters and RELU to the input image. Although we used various techniques to overcome the overfitting problem, it is possible that the current CNN model has over-fitted the data. Over-fitting could explain why the current CNN model with maximal information fails to significantly outperform a standard machine learning algorithm that uses the reduced summary information from catalogs. The best method to forestall over-fitting is to gather additional training pictures with spectroscopic development, as the execution of CNN enhances with all the more preparing information. However, spectroscopic perceptions are expensive and tedious, and it is hazy if adequate preparing information will be accessible in future photo-metric overviews. [9] If enough training data become available in DES, CNN's become an attractive option because it can be applied immediately on reduced, calibrated images to produce well-calibrated posterior probabilities. It is duly noted that using enormous and high resolution images will require the use of high powered multi-GPU systems. 


\section{B. Support Vector Machines in Morphological Galaxy clas- sification in Astronomy}

Morphological galaxy classification is a method used by astronomers to classify galaxies based on their structure and appearance. The below figure depicts one of the common classification schemes used in astronomy.

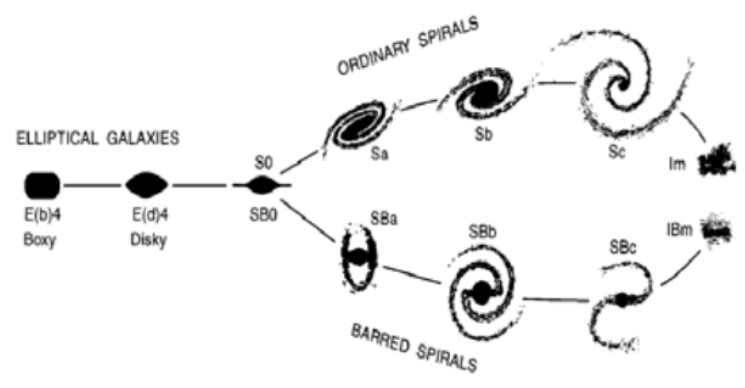

Figure 2. Morphological Galaxy Classification [10]

In order to classify a galaxy, [7] proceeded with extracting features from the images, On testing the Performance of the Classification Algorithms (PCA) using various combinations of input features on SVM with RBF kernel. The algorithm classifies galaxies into various classes, for instance, using only morphic features, only PCA features, and both morphic and PCA features combined. It then implements 10 -fold crossvalidation with the holdout images randomly selected from the overall training set (without replacement) at each iteration maximize the information provided by data set.

Since the training set differs at each iteration, PC vector is recalculated at every iteration and thus, a large amount of time is required. Performance of SVM is good because the features were not linearly separable even when projected to higher dimensions with the RBF kernel. [15]SVM's are popular in astronomy and have the advantage to work better with noise, have good predictive power, handles nonlinear functions approximations and are scalable. Unfortunately, this method have a tendency to over-fit and it requires a longer time to train the model and it is hard to interpret.

\section{Other Disadvantages in Current Algorithms used for Space Research}

1) The Nearest Neighbor algorithms don't require any training, it is easily parallelized and has good predictive power but they are computationally intensive and affected by noise and irrelevant properties. Astronomers prefer nearest neighbours for exoplanets search.

2) Expectation Maximization method has the advantage of fast convergence. It works with missing information as well. The disadvantage is that it is often simply biased toward Gaussian's and encounters the problem of local minima.

3) Artificial Neural Networks(ANNs) learn from training data set, so programmers do not need to mathematically describe the salient features. Unfortunately, as a result, ANNs require large numbers of data set to achieve high performance. Sifting through massive data sets to find a comprehensive number of examples to give to the code is cumbersome work, and it can defeat the entire purpose of trying to develop faster algorithms.

\section{Enhanced Current Methods using OTL AND ORGanic COMPUTING}

This section explains the different methods in Online transfer learning and organic computing.

\section{A. Transfer Learning}

[18]Transfer learning (TL) is an upcoming machine learning frameworks that has been actively studied recently. It works on the concept of knowledge transfer and builds models for target domain by extracting information from another existing source domain. Transfer learning is important for many applications where training data in a new domain may be limited or too expensive to collect. [6]Although transfer learning has been actively explored, most existing work on transfer learning was often studied in an offline learning fashion, which has to assume training data in the new domain is given a priori. Such an assumption may not always hold for some real applications where training examples may arrive in an online or sequential manner.

\section{B. Online Transfer Learning}

Online Transfer Learning is a machine learning framework where knowledge gets transferred between the source domain and target domain in real time, in order to help train a classifier of the target domain. Most machine learning techniques work on the assumption that the training and test data derive from similar source and distribution, if the distribution changes we need to start from scratch from newly collected data and learning tends to be more expensive. to overcome such issues, the TL model is introduced in machine learning. This reduces time and complexity and proven to be a cost-effective solution. The data distributions of the source Domains and target Domain can be very different.

[5]The OTL algorithm examines each instance in a sequential manner in real time. After observing the instance, it predicts the outcome based on its knowledge. The algorithm may receive feedback indicating correct output. The algorithm uses this feedback as knowledge gained from experience and improves itself for the future classification and better accuracy. OTL can be classified into two different sections based on the source domain and the target domain. They are Homogeneous Online Transfer Learning and Heterogeneous Online Transfer Learning.

1) Homogeneous Online Transfer Learning: In the homogeneous OTL, the source domain and the target domain have the same feature space.

The basic concept of this OTL solution is based on the ensemble learning approach. first, construct an entirely new prediction function $\mathrm{f}$ only from the data in the target domain in an online fashion, and then learn an ensemble prediction 
function that is the mixture of both the old and the new prediction functions which can transfer the knowledge from the source domain.

The disadvantage of using homogeneous transfer learning is that when the model training phase occurs, the prediction of the target variable is often biased and change over time. This phenomenon is known as concept drift.

[17]To eradicate the concept drift problem features both predictors from source and target domains are ensembled. The predictions of both the functions are combined using weights assigned to them. Updating the model involves a two-step framework. Initially, the prediction function (f), updated by using an online learning method which is the PA algorithm. The second step is to update the prediction weights dynamically based on the current weights and a function of the square loss of the prediction.

[21] Show that the use of multiple sources for a homogeneous OTL for single target domain results in faster and better accuracy. This concept can be adapted for multigalaxy classification with multiple type galaxy sources for an enhanced result and progress the space classification function.

2) Heterogeneous Online Transfer Learning: In Heterogeneous OTL, [2] assumed that the source and target domain are different. Heterogeneous OTL is generally very challenging. To simplify the problem, some assumptions are to be made such as the feature space of the source is a subset of the target domain. Due to the difference between the two feature spaces, it is not possible to directly apply the algorithm.

[2] proposes a multi-view approach for addressing the heterogeneous data problem as the source domain and target domain are very different and also assume that the first m-dimensions of the target dataset features represents the source dataset features. Each data instance is split into two, where the first part represents the source domain and the second part represents the new target domain. This helps the ensemble classifiers to classify the new observed data sample efficiently and forces the multi-view method not to deviate too much from the previous homogeneous OTL classifiers. The key idea of heterogeneous OTL is to adopt a co-regularization principle of online learning from two classifiers $f_{t}^{(1)}$ and $f_{t}^{(2)}$ simultaneously from two views and predict an unseen example on the target domain.

$$
\hat{y}_{t}=\operatorname{sign}\left(\frac{1}{2}\left(f_{t}^{(1)}\left(x_{2_{t}}^{(1)}\right)+f_{t}^{(2)}\left(x_{2_{t}}^{(2)}\right)\right)\right)
$$

\section{Organic Computing}

Organic computing is an emerging Research field that addresses the problems of organization in a complex system that can be tackled scientifically in a unified way.

[1]It is based on the facts that in future every human will be surrounded by autonomous systems. These are equipped with sensors and actuators and are aware of their environment. These systems can communicate freely, and organize themselves in order to perform the actions and services that seem to be required.

[33] The goal is to construct such systems as sturdy, safe, flexible, and trustworthy as possible. In specific, a strong orientation towards human needs, as opposed to a pure implementation of the technologically possible, seems absolutely central. In order to achieve these goals, our technical systems will have to act more independently, flexible, and autonomously, i.e. they will have to exhibit lifelike properties. We call such systems "organic". "Organic Computing System" is a technical system that adapts dynamically to exogenous and endogenous change. It is characterized by the properties of the organization, selfconfiguration, self-optimization, self-healing, self-protection, self-explaining, and context awareness.

\section{Enhancing Space Research and Astronomy Learning Methodology using Organic Computing and OTL}

This section we will see how we can use Organic Computing and OTL methods to enhance current Space Research and Astronomy Learning Algorithms.

1) Ensemble application of Organic Computing in Spacecraft: In the concept of Ensemble based organic computing systems, [27] consider a group of spacecraft for deep space missions where each individual unit of spacecraft could be autonomous but when viewed as a whole, they could be seen as a self-organized entity. [4]An organic computing system will respond dynamically to changes in the space and will also have sufficient freedom to do so.

Consider such a scenario for spacecraft in deep space as the basic entities, the application of organic computing methods and its benefits become clear. The future of transportation will be inter-galactic travel and it is expected that all vehicles will be interconnected and will communicate with each other to optimize travel times. In such scenarios, [24]the new domain will encounter incoming of rapid data from outer space when the spacecraft is traversing across the new planet or galaxy etc. To process such data instantaneously and learn from experience and adapting knowledge, organic computing principles need to be adapted. The adverse effects of any unintended scenario should be minimum, hence the system should selforganize accordingly. Organic computing helps in the area of navigation and safety for spacecraft by making the knowledge obtained transformed into a self-organizing mechanism. This concept is futuristic and requires advanced technologies that are not available today.

2) Self Learning of Maps and Navigation in Spacecraft: [3] [30] The concept of self-learning can be adapted to autonomous spacecraft. [11] Demonstrates the self-learning capabilities of Unmanned Aerial Vehicle(UAV) for maneuvering in the aerodromes. It considers the UAV to be present in a highly dynamic environment where there exists many foreign objects. [19] To address the obstacles in the path, a collection of two maps will be created and they are aerodrome map and the obstacle map. The aerodrome map shows the objects present visually in the path and course, and the obstacle map stores the learned obstacle distribution. The obstacle map can be combined with sensor array image to make it a robust 
obstacle map. The Bayesian approach handles the self-learning part. The bayesian inference is applied to the aerodrome map and the obstacle map separately. In this concept, the probability of obstacles obtained from images are considered and its uncertainty level is calculated. Higher the uncertainty value, more relevance is placed on the aerodrome map. Using this method, if an object repeatedly appears in the images, it will be confirmed and learned with increasing confidence. The confidence measure is an advantage to the Bayesian method as the confidence measure cannot be obtained through normal computer vision algorithms. We can adopt this concept of selflearning of visual maps to spacecraft, where it can traverse its course in space.

3) Multi Source Domain Knowledge Extraction for Astronomy: In this concept, [22] [26] the knowledge obtained from multiple source domains and transferred to a single target domain. [14] Assert that by building a model from multiple homogeneous source domains for knowledge transfer to the final model becomes more refined. The model learns to identifies elements of core knowledge and features that are to be transferred to the target domain.

[31]This approach can be implemented in heterogeneous OTL, where the target domain feature space will be split into two sets, the first set has the homogeneous features that are common to both the source and target domains and the second set will contain heterogeneous features.

[14] An image classification example is used to obtain the features of the target images from multiple source domains and transfer the source knowledge to a new model. It combines multiple classifiers created from the source domains to form an ensemble classifier for the target domain. The source domain data is given in advance and so for each source domain, a classifier is built in an offline learning paradigm. The target data arrives in a sequential manner in real time. [32] PA is used for learning the representation of the target data. The hinge loss function calculates the decision loss and adds it to prior learned samples. In heterogeneous transfer learning scenario, the feature space will be split into two.

The first part is assumed to be homogeneous with the source domain while the second part is heterogeneous with the source domain. A set of three base classifiers $f_{s}^{(i)}, f_{t_{1}}^{(i, t)}, f_{t_{2}}^{(i, t)}$ are learned for each source domain", , where $f_{s}^{(i)}$ is the source domain classifier and $f_{t_{1}}^{(i, t)}, f_{t_{2}}^{(i, t)}$ are the target domain classifiers. $f_{t_{1}}^{(i, t)}$ and $f_{t_{2}}^{(i, t)}$ are learned by combining the first section and the second section in target domain with the source domain, respectively [14]. In the next step, the weights pertaining to each classifier is learned. [29] The combination of the base classifiers and learned weights produces a robust classifier that can perform well in the target domain.

This concept can be used in morphological galaxy classification to determine the various features and classes of galaxies. Multiple galaxy images and data set can be considered as sources to determine an unknown target galaxy and enhance its knowledge.
4) Combining Convolutional Neural Network and Online Transfer Learning for Meteors and Exoplanets Tracking: [7]A neural network method is proposed where it learns to discriminate features of an object and tracks the location and size of the object. It utilizes the merits of single object trackers in adapting appearance models and searching for a target in the next frame. The learning process comprised of transfer learning and object tracking is done in an online learning framework. [25]select a deep neural network because of the capability of the network of learning high-level feature representations of targets. The key idea of the authors for this experiment is to use the CNN layers of the deep neural network as a generic and middle-level image representation. The neural network is trained on the given training data set. After the training the model, with the exception of a fully connected layer, every other layer gets transferred to the target task. [23]The network is then trained on the Multiple Object Tracking(MOT) dataset of the object tracking task.

Particle Filtering Network does the object tracking which implements the Bayesian filter. The particle filter has two main components. They are

1. The dynamic model which generates candidate samples based on prior experience

2. Observation model computes the similarity between the prediction and the actual value.

From the earlier trained neural network where the output neuron gives out a score and its likelihood value is computed. The likelihood is calculated by the equation.

$$
p\left(y_{t} \mid x_{t}\right)=\exp \left(d_{t}\right)
$$

This concept is a combination of CNN with OTL can be adapted for meteor and exoplanet tracking in deep space. When tracking an object, the appearance of the object might change due to its motion. To address this, the likelihood function needs to adapt over time by fine-tuning the neural network model. This problem exists in OTL as concept drift and can be rectified as well. This issue can be addressed using predictions method explained above in Homogeneous OTL.

\section{E. Disadvantages and Problems in OTL and Neural Net- works}

- Concept Drift needs to resolve effectively. Although PA resolves concept drift to some extent, to completely eradicate the issue, few measures need to be taken.

OTL may suffer from a negative transfer of knowledge when facing a serious concept drifting problem. Some of the measures are :

1) Periodically re-fitting the datasets.

2) Regularly updating the learning models.

3) Observe and learn from the change in the pattern of the data.

4) Data preparation to remove the systematic changes to data over time.

- OTL techniques usually produce denser classifiers and take a lot of time to process. This is unavoidable as the 
OTL algorithms make use of the old classifier from the source domain.

- Poison Frogs attacks need to be addressed. [12] Poison frogs are targeted clean-label poisoning methods that attack at training time with the goal of manipulating testtime behavior. These attacks are difficult to detect because they involve non-suspicious (correctly labeled) training data, and do not degrade the performance on non-targeted examples.

OTL and neural networks use label data for training the models and knowledge gets transferred to the target domain using these label tags. In the domain of astronomy and deep space, targeted attacks are imminent and attacks are like poison frogs needs to be addressed to avoid the data manipulation issues. It is duly noted that many neural networks are trained using data sources that are easily manipulated by adversaries.

\section{Conclusions}

We have outlined some of the current methods used in deep space exploration of planets and galaxies and concepts of OTL and Organic Computing that can be adapted to enhance the Deep Space Research. It shows how different concepts and methods that can be infused together to work in tandem that would result in better performance and output than individual methods achieved. Organic Computing and OTL is able to address some of the problems in a dynamic environment change by adapting trained knowledge transfer to target domains and self-learning methodologies.Some of the proposed methods are futuristic concepts and requires more advanced Machine learning models.

The paper also tells the drawbacks in the current methods as well as in OTL methods which we hope to encourage the investigation of the issue and address the harder problems. We believe that organic computing and OTL can be one of the better options that could be adapted to enhance the space research programs and astronomy for the betterment of mankind.

\section{REFERENCES}

[1] C. Muller-Schloer and S. Tomforde, " Organic Computing-Technical Systems for Survival in the Real World. Springer, 2017.

[2] Peilin Zhao and Steven C.H. Hoi, "OTL: A Framework of Online Transfer Learning".Artificial Intelligence. 216, 76-102. Research Collection School Of Information Systems.

[3] Christofer Fellicious,"Transfer Learning and Organic Computing for Autonomous Vehicles",arXiv:1808.05443v1 [cs.LG] 16 Aug 2018

[4] Xu Lan, Xiatian Zhu, and Shaogang Gong "Knowledge Distillation by On-the-Fly Native Ensemble,arXiv:1806.04606v2 [cs.CV] 8 Sep 2018

[5] Zhaodong Wang and Matthew E. Taylor,'Interactive Reinforcement Learning with Dynamic Reuse of Prior Knowledge",arXiv:1805.04493v1 [cs.AI] 11 May 2018

[6] Jianfei Yu, Minghui Qiu, Jing Jiang, Jun Huang, Shuangyong Song, Wei Chu, Haiqing Chen"Modelling Domain Relationships for Transfer Learning on Retrieval-based Question Answering Systems in Ecommerce",arXiv:1711.08726v1 [cs.CL] 23 Nov 2017

[7] Qi Chu1,3 , Wanli Ouyang2,3 , Hongsheng Li3 , Xiaogang Wang3 , Bin Liu1, Nenghai Yu1,Online Multi-Object Tracking Using CNN-based Single Object Tracker with Spatial-Temporal Attention Mechanism, arxiv/1708.028432017
[8] Amy McGovern · Kiri L. Wagstaff, Machine learning in space: extending our reach, Springer, 2017

[9] Onboard object recognition for planetary exploration,Burl, M.C. \& Wetzler, P.G. Mach Learn(2011) 84: 341: doi.org/10.1007/s10994-011$5239-6$

[10] Siddhartha Kasivajhula, Naren Raghavan, and Hemal Shah, Morphological Galaxy Classification Using Machine Learning, citeseerx.ist.psu.edu

[11] Himanshu S. Bhatt, Arun Rajkumar and Shourya Roy, Multi-Source Iterative Adaptation for Cross-Domain Classification, Xerox Research Centre India

[12] W.Ronny Huang,Ali Shafahi,Mahyar Najibi,Octavian Suciu,Christoph Studer,Tudor Dumitras, Poison Frogs! Targeted Clean-Label Poisoning Attacks on Neural Networks, arXiv:1804.00792v2

[13] Nour Eldeen M. Khalifa1, Mohamed Hamed N. Taha1, Aboul Ella Hassanien1, I. M. Selim, Deep Galaxy: Classification of Galaxies based on Deep Convolutional Neural Networks, arxiv:1709.02245

[14] Edward J. Kim, Robert J. Brunner; Star-galaxy classification using deep convolutional neural networks, Monthly Notices of the Royal Astronomical Society, Volume 464, Issue 4, 1 February 2017, Pages 4463-4475

[15] Durgesh K. Srivatsa,Lekha Bhambu,DATA CLASSIFICATION USING SUPPORT VECTOR MACHINE,Journal of Theoretical and Applied Information Technology

[16] Koby Crammer,Ofer Dekel, Joseph Keshet, Shai Shalev-Shwartz , Yoram Singer,Online Passive-Aggressive Algorithms,Journal of Machine Learning Research 7 (2006) 551-585,

[17] Jason Brownlee, A Gentle Introduction to Concept Drift in Machine Learning, Machine Learning Mastery. 2018

[18] A Survey on Transfer Learning, Sinno Jialin Pan and Qiang Yang,IEEE, 2008

[19] Kernel Embedding Approaches to Orbit Determination of Spacecraft Clusters Srinagesh Sharma, James W. Cutler, arXiv:1803.00650, 2018

[20] I. Nigam, C. Huang, and D. Ramanan, "Ensemble knowledge transfer for semantic segmentation," in 2018 IEEE Winter Conference on Applications of Computer Vision (WACV). IEEE, 2018, pp. 1499-1508.

[21] Wu et al., "Online Transfer Learning with Multiple Homogeneous or Heterogeneous Sources," in IEEE Transactions on Knowledge and Data Engineering, vol. 29, no. 7, pp. 1494-1507, 1 July 2017. doi: 10.1109/TKDE.2017.2685597

[22] A Survey on Online Transfer Learning With Multiple Homogeneous or Heterogeneous Sources, Ashish P. Gaigol, Dr.Himanshu U. Joshi, IJIRSET,2017

[23] Astronomical Image Reconstruction with Convolutional Neural Networks,remi Flamary,arXiv:1612.04526v2 [cs.CV] 7 Jun 2017

[24] Deep Transfer Learning for Cross-domain Activity Recognition, Jindong Wang, Vincent W.Zheng, arXiv:1807.07963v2 [eess.IV] 19 Aug 2018

[25] Online Transfer Learning in Reinforcement Learning Domains Yusen Zhan, Matthew E. Taylor,AAAI 2015 Fall Symposium

[26] Online Transfer Learning with Multiple Homogeneous or Heterogeneous Sources Qingyao Wu, Hanrui Wu, Xiaoming Zhou, Mingkui Tan, Yonghui $\mathrm{Xu}$, Yuguang Yan, and Tianyong Hao,IEEE transactions on data and knowledge, vol. 29, no. 7, july 2017

[27] Transfer Learning for Multiple-Domain Sentiment Analysis - Identifying Domain Dependent/Independent Word Polarity, Yasuhisa Yoshida, Tsutomu Hirao,Tomoharu Iwata, Masaaki Nagata,Yuji Matsumoto $\dagger$, Twenty-Fifth AAAI Conference on Artificial Intelligence

[28] Machine learning algorithms in Astronomy, E M Howard,ADASS XXV: The 25th Annual ADASS Conference.

[29] Metalearning for multiple-domain Transfer Learning,Catarina Felix Carlos Soare and Alipio Jorge,http://ceur-ws.org/Vol-1455

[30] B. Lu, M. Coombes, B. Li, and W. H. Chen, "Improved situation awareness for autonomous taxiing through self-learning," IEEE Transactions on Intelligent Transportation Systems, vol. 17, no. 12, pp. 3553-3564, Dec 2016.

[31] J. T. Zhou, S. J. Pan, I. W. Tsang, and Y. Yan, "Hybrid heterogeneous transfer learning through deep learning." in AAAI, 2014, pp. 2213-2220.

[32] S. Moon and J. Carbonell, "Completely heterogeneous transfer learning with attention-what and what not to transfer," in Proceedings of the 26th International Joint Conference on Artificial Intelligence. AAAI Press, 2017, pp. 2508-2514.

[33] Organic Computing, https://en.wikipedia.org/wiki/Organic-computing 